\title{
Umfrageergebnisse
}

\section{Telematik und Telemedizin überzeugen Ärzte grundsätzlich}

\section{Der erste E-Health-Report der Bundesärztekammer zeigt es deut- lich: Ärzte sehen die Vorteile der Telematik. Vielen Niedergelassenen ist die praktische Relevanz des Themenkomplexes samt seiner Optionen aber noch fern.}

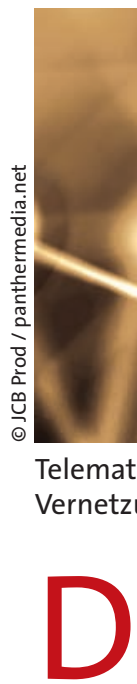

er 111. Ärztetag im Jahr 2008 in Ulm war in Sachen elektronischer Gesundheitskarte sicher eine Sternstunde in der Geschichte des Gremiums. Mehrere Stunden lang wurde über die Telematik inklusive elektronischer Gesundheitskarte (eGK) äußerst kontrovers diskutiert. Damals beauftragte der Deutsche Ärztetag die Bundesärztekammer (B̈̈K) mit der Erhebung repräsentativer Daten über die Haltung der deutschen Ärzte zu Telematik und Telemedizin. Diese Daten, vom Allensbach Institut im April 2010 bei knapp 600 Ärzten repräsentativ erhoben, liegen nun in Form der ersten großen E-Health-Umfrage der BÄK vor.

Die Antworten auf die teilweise sehr detailliert gestellten Fragen zeigen deutlich, wie differenziert Ärzte an die elektronische Vernetzung herangehen. Beispiel Telemedizin: $80 \%$ sehen einen großen oder sehr großen Nutzen der Teleradiologie. Bei Telekonsultationen, wie sie teilweise heute schon bei der Versorgung akuter Schlaganfallpatienten eingesetzt werden, sind es $63 \%$. Beim ambu- lanten Telemonitoring liegt die Quote bei $54 \%$.

Differenziert wird auch bei der Bewertung der Telematikanwendungen. „Während $76 \%$ der befragten Ärzte einen großen oder sehr großen Nutzen der elektronischen Speicherung von Notfalldaten konstatieren, halten nur $37 \%$ das elektronische Rezept für sehr nützlich oder nützlich“, betonte Dr. Franz-Joseph Bartmann von der BÄK. Dies zeige deutlich, dass die Prioritäten der Ärzte bei den medizinischen Anwendungen lägen. Elektronischer Arztbrief, Arzneimitteltherapiesicherheitsprüfung und elektronische Patientenakte liegen im guten Mittelfeld: Zwischen $60 \%$ und $69 \%$ votierten hier für „(sehr) nützlich“.

\section{Klinikärzte bislang aufgeschlossener}

Frappierend ist ein erheblicher Unterschied zwischen ambulant und stationär tätigen Ärzten. Durch die Bank stehen Klinikärzte den Telematikanwendungen sehr viel aufgeschlossener gegenüber. Jeder fünfte niedergelassene Arzt sagt sogar, dass eeine Telematikanwendung überhaupt keine große Verbesserung sei. Im Krankenhaus sagt das praktisch niemand.

„Karl Marx hatte Recht: Das Sein bestimmt das Bewusstsein“, kommentierte Bartmann diese Daten. Das Problem der Verfügbarkeit von Befunden sei im stationären Bereich eben wesentlich ausgeprägter als im ambulanten, wo Patienten ihren Hausarzt selten wechseln und Fachärzte meist anlassbezogen hinzugezogen würden. BÄK-Vizepräsident Dr. Frank Ulrich Montgomery wies zudem darauf hin, dass Krankenhausärzte schon heute sehr viel stärker in Kontakt mit Dingen wie elektronischen Patientenakten oder Teleradiologie kämen. Mit der Nutzung im Alltag verschwinde die Skepsis oft rasch, so Montgomery.

Nicht ganz so ausgeprägt sind die Meinungsverschiedenheiten bei der Telemedizin: Hier sagen $88 \%$ der Klinikärzte und $64 \%$ der Niedergelassenen, dass die Vorteile die Nachteile überwögen. Dass die Telemedizin in der eigenen Praxis in Zukunft eine Rolle spielen werde, glauben immerhin $48 \%$ der niedergelassenen Ärzte. Im Krankenhaus sind es $80 \%$.

Montgomery wertete das Umfrageergebnis als Auftrag an die BÄK, weiterhin konstruktiv, aber immer kritisch am Aufbau der Telematikinfrastruktur und an der Arbeit der gematik mitzuwirken: „Wir werden peinlichst darauf achten, dass die Ärztetagsbeschlüsse zur Telematik adäquat umgesetzt werden“, sagte er. Vor allem beim Thema elektronische Patientenakten sei Wachsamkeit geboten. Nicht nur müssten die Daten sicher sein. Sie dürften auch keinesfalls in der Hoheit von Kostenträgern gespeichert werden. „Elektronische Patientenakten gehören in die Hand des Hausarztes, des Patienten und gegebenenfalls des behandelnden Arztes in Klinik und Praxis." gvg

Die Befragung im Internet: www.baek.de/ downloads/eHealth_Bericht_lang_final.pdf 